\title{
Complications of Covid-19: A Systematic Review and Meta-Analysis
}

Aishat Temitope Alonge, Babatunde Isaac Ademusire, Chinweoge Frances Epum, Boluwatife Adeleye Adewale, Opeoluwa Samuel Adefarati

College of Medicine, University of Ibadan, Nigeria

\begin{abstract}
Objectives: COVID-19, primarily a respiratory disease, can have complications that affect all organ systems of the body. There is a paucity of systematic reviews on all the complications. In this systematic review and meta-analysis, we set out to summarize the complications of COVID-19 in all body systems and their prevalence.
\end{abstract}

Methods: PubMed and Google Scholar were searched for eligible articles using predefined criteria. Database searching and extraction were performed by independent reviewers.

Results: We identified 74 case reports/series and 15 observational studies. In both the case reports/series and observational studies, pulmonary complications were the most commonly reported, particularly pneumonia, followed by neurological complications in case reports/case series and hematological complications in observational studies. Atrial arrhythmias (1.7\%) and acute myopericarditis (1.7\%), liver injury (3.3\%), acute kidney injury (8.8\%), deep venous thrombosis $(2.2 \%)$, ischemic stroke (12.2\%), herpes zoster (1.1\%), and diabetic ketoacidosis (1.1\%) were the most reported cardiovascular, gastrointestinal, renal, hematological, neurological, dermatological, and endocrine complications respectively in case reports/series. However, acute myocarditis (100\%), hypoproteinemia (15.9-28.8\%), transient acute renal failure (49.9-90.1\%), acute coagulopathy (16.5-28.4\%), and ischemic stroke (1.3-3.9\%) had the highest pooled prevalence for cardiovascular, gastrointestinal, renal, hematological, and neurological complications respectively in observational studies.

Conclusion: The complications of COVID-19 are multi-systemic with pulmonary complications being the most commonly reported. Notwithstanding, healthcare professionals should be aware that COVID-19 is a differential diagnosis for even the rare but equally debilitating complications and should screen patients who develop these complications to rule out COVID-19 during the pandemic and beyond. J Microbiol Infect Dis 2021; 11(2): 45-57.

Keywords: Covid-19, SARS-CoV-2, Complications, Sequelae, Systematic review

\section{INTRODUCTION}

Coronavirus disease 2019 (COVID-19) is a respiratory disease that was firstly recorded in Wuhan China as a group of unexplained pneumonia cases and has since then become a global pandemic [1]. Coronaviruses are transmitted between humans and animals and commonly presents in humans with symptoms like fever, headache, fatigue, sore throat, cough, breathlessness, and some other common respiratory symptoms [2-4].

It has been shown from various studies that COVID-19 affects all the systems of the human body and this may be due to either weakening of the immune system caused by the infection or by replication and dissemination of the virus outside the lungs [5]. Therefore, we conducted a systemic review of the complications of COVID19 by analyzing and summarizing different case reports/series and observational studies.

\section{METHODS}

\section{Inclusion and exclusion criteria}

We conducted this systematic review following the Preferred Reporting Items for Systematic and Meta-analysis (PRISMA) protocols and guidelines. We included studies (case reports, case series, and observational studies) reporting 
complications seen in COVID-19 patients during the pandemic. We excluded studies that did not report the complications seen in COVID-19 patients. Systematic reviews, articles that are abstracts-only or without full text, non-English articles, and articles not freely available were also excluded.

\section{Outcomes of interest}

The primary outcome was to identify all the complications of COVID-19 among infected patients during the COVID-19 pandemic. We also aimed to report the prevalence of these complications.

\section{Search strategy}

Database searching was carried out by two reviewers independently (BIA and CFE) from July 1, 2020, till July 17, 2020. A comprehensive initial search was carried out using PubMed and Google Scholar. The PubMed database was searched using the following terms: (COVID-19 OR coronavirus OR SARS-COV-2) AND (symptom[Title] OR sign[Title] OR clinical characteristics[Title] OR clinical feature[Title]) and (COVID-19 OR coronavirus or SARS-COV2) AND (Complication[Title/Abstract]). Related titles and abstracts were subsequently screened to select studies that met the inclusion criteria. Eligible articles were further screened by applying the predefined inclusion and exclusion criteria. Manual searching was done by ATA, $\mathrm{BAA}$, and OSA using the reference lists, citation lists, and related articles of all the eligible articles. The last publishing date of the article included in this study is July 16, 2020. All articles identified had already been included from the first search. Figure 1 shows the flow diagram of the screening process of studies included in this systematic review and metaanalysis.

\section{Data extraction}

The data from included studies were extracted using a customized template and entered into Microsoft Excel by two autonomous reviewers (BIA and CFE). A third reviewer (ATA) resolved the differences between the two autonomous authors. The following data were extracted: authors' names, year of publication, journal, country of patient recruited, year of recruitment, year of research, the population of the study, sample size, follow up duration, study design, sample size, respiratory complications, cardiovascular complications, gastrointestinal complications, renal complications, hematological complications, neurological complications, dermatological complications, and endocrine complications. Complications that do not strictly fit into a particular body or functional system were classified as others. The number of reported complications in various organ systems was entered for each study.

\section{Statistical analysis}

Data was exported into Statistical Package for the Social Sciences (SPSS) version 23 for analysis - case report/series and observational studies separately. Counts and proportions were computed for each complication. The proportion for the observational studies was computed as the number of positive findings relative to the total sample size of studies that reported the complication. The $95 \%$ confidence intervals of proportions reported in the observational studies were determined using an online calculator [6].

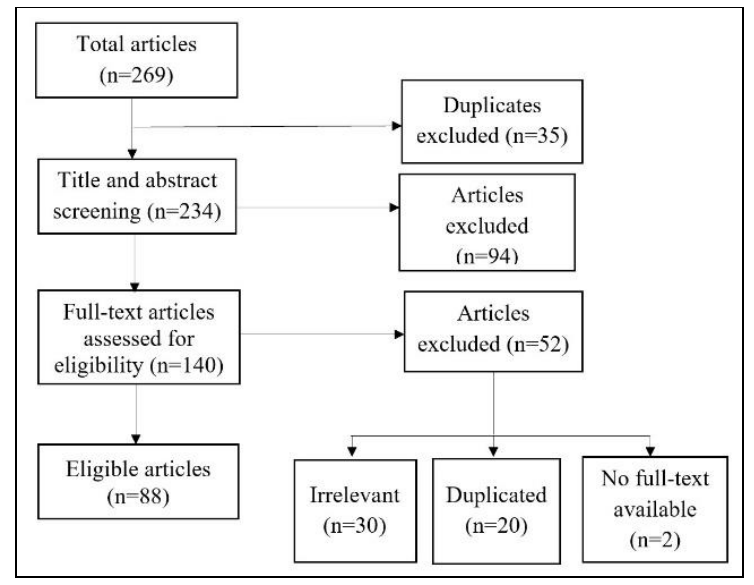

Figure 1. Flow diagram showing the screening process of studies included in this systematic review.

\section{RESULTS}

Eighty-eight papers published in 2020 were included in this systematic review; 51 case reports, 22 case series, 2 prospective studies, and 12 retrospective studies; 1 consisted of both a case-control study and case series. Only 3 of the studies had an experimental and control group in the design, while the rest were purely experimental groups. The majority (25) of the studies had patients recruited from the United States, 14 from Italy, 12 studies recruited from the United Kingdom, and 11 from China. The largest study so far is a prospective cohort study - with patients recruited from 24 countries - on 
pulmonary complications and mortality of COVID-19.

From the 74 published case reports/series, 38 reported pulmonary complications, 14 reported cardiovascular complications, 3 reported gastrointestinal complications, 8 reported renal complications, 10 reported hematological complications, 24 reported neurological complications, 2 reported dermatological complications, and 2 reported endocrine complications.

Among the observational studies, 10 studies reported pulmonary complications, 6 cardiovascular, 4 gastrointestinal, 6 renal, 6 hematological, and 2 neurological complications. Details on the included studies and the reported complications could be seen in the Additional File 1. The pooled prevalence estimates from the observational studies show that pneumonia (41.4\%; 95\% Cl: 38.6 - 44.1\%) and acute respiratory distress syndrome (ARDS) (22.2\%; $95 \% \mathrm{Cl}: 20.2$ - 24.1\%) were the commonest respiratory complications (Table 1). There were 5 cases given a diagnosis of COVID-19 associated pulmonary aspergillosis (CAPA) and 4 cases of Aspergillosis colonization.

The pooled prevalence estimates of common complications associated with COVID-19 were $25.6 \%(95 \% \mathrm{Cl}: 25.6$ - 41.0\%) for myocardial injury and $20.0 \%$ (95\% Cl: 5.7 - 43.7\%) for pericardial effusion (Table 2). Acute coagulopathy (22.5\%; 95\% Cl: 16.5 - 28.4\%) was the commonest hematological complication associated with COVID-19 (Table 3). There were only 2 observational studies that reported neurological complications, reporting low prevalence of ischemic stroke $(2.3 \% ; 95 \% \mathrm{Cl}$ : $1.3-3.9 \%$ ), seizures (0.47\%; 95\% Cl: $0.01-$ $2.60 \%)$ and hemorrhagic stroke $(2.3 \% ; 95 \% \mathrm{Cl}$ : $0.8-5.5 \%$ ) (Table 4). Of the 51 case reports and 23 case series published with 181 patients in total, there were 121 (67\%) males and 60 (33\%) females. Pulmonary complications were most commonly reported, followed by neurological, then renal complications. Pneumonia was the commonest respiratory complication (Table 1), whereas ischemic stroke was the commonest neurological complication (Table 4).

There were 1,830 reported complications reported from the 15 observational studies included in this review. Pulmonary complications constituted the majority $(65.7 \%)$ of the reported complications, followed by hematological complications (8.1\%). There were 101 (5.5\%) cardiovascular complications (Table 2), 97 (5.3\%) gastrointestinal complications, 87 (4.8\%) renal complications (Table 5) and 21 (1.1\%) neurological complications (Table 4).

Other commonly reported complications (Table 6) include transient acute renal failure (70.0; 95\% Cl: 49.9 - 90.1\%), hypoproteinemia (22.4\%; 95\% Cl: 15.9 - 28.8\%) and electrolyte disorders (29.8\%; 95\% Cl: 22.8-36.9\%).

\section{DISCUSSION}

\section{Respiratory complications}

COVID-19 causes more damage to the respiratory system more than any other system. The respiratory route is the main route of entry of severe acute respiratory syndrome coronavirus 2 (SARS-CoV-2) as angiotensinconverting enzyme 2 (ACE2) receptors are well expressed on the lung alveolar epithelial cells. These receptor proteins are also found in the gastrointestinal system and the venous and arterial endothelium which are probably responsible for vascular and hematological complications [7].

Our findings reveal that about 4 in 10 hospitalized COVID-19 cases have pneumonia (Table 1) commonly presenting with fever, cough, and dyspnea. The risk factors for this level of illness severity in COVID-19 patients include old age, immunosuppression, diabetes mellitus, and cardiovascular and lung comorbidities [8]. Similar to influenza virusinduced pneumonia, the condition can be further complicated by acute respiratory distress syndrome (ARDS). However, ARDS in COVID19 can be somewhat atypical with a later onset time (8-12 days) than stipulated by Berlin criteria (1 week) [9]. COVID-19 induced ARDS patients may in fact have normal lung compliance [10].

These complications progress to respiratory failure which is the most likely cause of COVID19 mortality. Invasive Aspergillosis must be excluded if antibacterial therapy becomes ineffective and disease is rapidly advancing, particularly in immunocompromised patients [11]. 
Table 1. Respiratory complications of COVID-19.

\begin{tabular}{|c|c|c|c|c|}
\hline \multirow{2}{*}{ Respiratory Complications, $\mathrm{n}=107$ (59.1) } & \multirow{2}{*}{$\begin{array}{c}\text { Case reports/series }(N=181) \\
n(\%)\end{array}$} & \multicolumn{3}{|c|}{ Observational studies } \\
\hline & & Number of studies reporting complication & $\mathrm{n} / \mathrm{N}(\%)^{*}$ & $95 \%$ Confidence Interval $(\mathrm{Cl})$ \\
\hline Acute respiratory distress syndrome & $4(2.2)$ & 6 & $387 / 1747(22.2)$ & $20.2-24.1$ \\
\hline Pneumothorax & $8(4.4)$ & 2 & $2 / 253(0.8)$ & $0.1-2.9$ \\
\hline Invasive Aspergillosis & $1(0.6)$ & 1 & $9 / 147(6.1)$ & $2.8-11.6$ \\
\hline Respiratory failure only & $6(3.3)$ & 1 & 6/161 (3.7) & $1.4-8.1$ \\
\hline Pneumonia & $36(19.9)$ & 3 & $501 / 1211(41.4)$ & $38.6-44.1$ \\
\hline Unexpected ventilation & & 1 & $240 / 1128(21.3)$ & $18.9-23.7$ \\
\hline Pulmonary embolism & $21(11.6)$ & 4 & $58 / 2190(2.6)$ & $2.0-3.4$ \\
\hline Pleural effusion & $3(1.7)$ & & & \\
\hline Acute chest syndrome & $2(1.1)$ & & & \\
\hline Mediastinal emphysema & $1(0.6)$ & & & \\
\hline Giant bulla & $2(1.1)$ & & & \\
\hline Pneumomediastinum & $12(6.6)$ & & & \\
\hline Subcutaneous emphysema & $6(3.3)$ & & & \\
\hline Hydropneumothorax & $1(0.6)$ & & & \\
\hline Pulmonary capillary leak syndrome & $2(1.1)$ & & & \\
\hline Diffuse alveolar hemorrhage & $2(1.1)$ & & & \\
\hline
\end{tabular}


Table 2. Cardiovascular complications of COVID-19.

\begin{tabular}{|c|c|c|c|c|}
\hline \multirow{2}{*}{ Cardiovascular Complications, $\mathrm{n}=17$ (9.3) } & \multirow{2}{*}{$\begin{array}{l}\begin{array}{l}\text { Case reports/series } \\
(\mathrm{N}=181)\end{array} \\
\mathrm{n}(\%)\end{array}$} & \multicolumn{3}{|c|}{ Observational studies } \\
\hline & & Number of studies reporting complication & $\mathrm{n} / \mathrm{N}(\%)^{*}$ & 95\% Confidence Interval (Cl) \\
\hline Myocardial injury & $1(0.6)$ & 2 & $48 / 144(33.3)$ & $25.6-41.0$ \\
\hline Heart failure only & & 1 & $9 / 161(5.6)$ & $2.6-10.6$ \\
\hline Malignant arrhythmia & & 1 & 3/161 (1.9) & $0.4-5.4$ \\
\hline Myocardial infarction & $1(0.6)$ & 2 & $6 / 549(1.1)$ & $0.4-2.4$ \\
\hline Ventricular tachycardia/ Ventricular fibrillation & & 1 & $11 / 187(5.9)$ & $2.9-10.5$ \\
\hline Acute myocarditis & $2(1.1)$ & 1 & $20 / 20(100)$ & 100 \\
\hline Pericardial effusion & $1(0.6)$ & 1 & $4 / 20(20.0)$ & $5.7-43.7$ \\
\hline Acute effusive pericarditis with cardiac tamponade & $1(0.6)$ & & & \\
\hline Intramural hematoma & $1(0.6)$ & & & \\
\hline Atrial arrhythmia & $3(1.7)$ & & & \\
\hline Acute myopericarditis & $3(1.7)$ & & & \\
\hline Acute thrombosis of aortic prosthetic graft & $1(0.6)$ & & & \\
\hline High degree atrioventricular block & $1(0.6)$ & & & \\
\hline Left ventricular thrombus & $1(0.6)$ & & & \\
\hline Takotsubo cardiomyopathy & $1(0.6)$ & & & \\
\hline
\end{tabular}

*pooled prevalence of complications in the observational studies expressed as the number of reported complications divided by the total sample size of studies reporting the complication Table 3. Hematological complications of COVID-19.

J Microbiol Infect Dis $\quad$ www.jmidonline.org $\quad$ Vol 11, No 2, June 2021 


\begin{tabular}{|c|c|c|c|c|}
\hline \multirow{2}{*}{ Hematological Complications, $n=16$ (8.8) } & \multirow{2}{*}{$\begin{array}{l}\text { Case reports/series } \\
\qquad \begin{array}{c}(\mathrm{N}=181) \\
\mathrm{n}(\%)\end{array}\end{array}$} & \multicolumn{3}{|c|}{ Observational studies } \\
\hline & & Number of studies reporting complication & $\mathrm{n} / \mathrm{N}(\%)^{*}$ & 95\% Confidence Interval $(\mathrm{Cl})$ \\
\hline Acute coagulopathy & & 1 & $42 / 187(22.5)$ & $16.5-28.4$ \\
\hline Deep venous thrombosis & $4(2.2)$ & 4 & $27 / 1144(2.4)$ & $1.6-3.4$ \\
\hline Venous abdominal thrombosis & $1(0.6)$ & & & \\
\hline Arterial abdominal thrombosis & $3(1.7)$ & 1 & $11 / 400(2.8)$ & $1.4-4.9$ \\
\hline Disseminated intravascular coagulation & $1(0.6)$ & 3 & $39 / 820(4.8)$ & $3.4-6.5$ \\
\hline Bleeding events & $4(2.2)$ & 1 & $19 / 400(4.7)$ & $2.9-7.4$ \\
\hline Non-vessel thrombotic complication & & 1 & $8 / 400(2.0)$ & $0.9-3.9$ \\
\hline Superficial venous thrombosis & & 1 & $2 / 400(0.50)$ & $0.06-1.81$ \\
\hline Methemoglobinaemia in glucose-6-phospate deficiency & $1(0.6)$ & & & \\
\hline Vasocclusive crisis & $2(1.1)$ & & & \\
\hline
\end{tabular}

*pooled prevalence of complications in the observational studies expressed as the number of reported complications divided by the total sample size of studies reporting the complication

Table 4. Neurological complications of COVID-19.

J Microbiol Infect Dis $\quad$ www.jmidonline.org $\quad$ Vol 11, No 2, June 2021




\begin{tabular}{|c|c|c|c|c|}
\hline \multirow{2}{*}{ Neurological Complications, $n=82$ (45.3) } & \multicolumn{2}{|l|}{ Case reports/series $(\mathrm{N}=181)$} & \multicolumn{2}{|c|}{ Observational studies } \\
\hline & $\mathrm{n}(\%)$ & $\begin{array}{l}\text { Number of studies } \\
\text { reporting complication }\end{array}$ & $\mathrm{n} / \mathrm{N}(\%)^{*}$ & 95\% Confidence Interval $(\mathrm{Cl})$ \\
\hline Ischemic stroke & $22(12.2)$ & 2 & $14 / 602(2.3)$ & $1.3-3.9$ \\
\hline Guillain-Barre syndrome (GBS) & $12(6.6)$ & & & \\
\hline Basal ganglia involvement & $1(0.6)$ & & & \\
\hline Rhombencephalitis & $1(0.6)$ & & & \\
\hline Myasthenia Gravis crisis & $1(0.6)$ & & & \\
\hline Encephalopathy & $2(1.1)$ & & & \\
\hline Phrenic nerve paralysis & $1(0.6)$ & & & \\
\hline Cerebral vasculitis & $2(1.1)$ & & & \\
\hline Subarachnoid hemorrhage & $5(2.8)$ & & & \\
\hline Posterior reversible encephalopathy syndrome & $3(1.7)$ & & & \\
\hline Polyneuritis cranialis & $1(0.6)$ & & & \\
\hline Intracerebral hemorrhage & $7(3.9)$ & 1 & $1 / 214(0.47)$ & $0.01-2.60$ \\
\hline Seizures & $13(7.2)$ & 1 & $1 / 214(0.47)$ & $0.01-2.60$ \\
\hline Hypoxic ischemic brain injury & $7(3.9)$ & & & \\
\hline Transient ischemic attack & $1(0.6)$ & & & \\
\hline Hemorrhagic stroke & $3(1.7)$ & 1 & $5 / 214(2.3)$ & $0.8-5.5$ \\
\hline
\end{tabular}

*pooled prevalence of complications in the observational studies expressed as the number of reported complications divided by the total sample size of studies reporting the complication Table 5: Gastrointestinal, renal, and dermatological complications of COVID-19.

J Microbiol Infect Dis $\quad$ www.jmidonline.org $\quad$ Vol 11, No 2, June 2021




\begin{tabular}{|c|c|c|c|c|}
\hline \multirow{2}{*}{ Complications } & \multirow{2}{*}{$\frac{\text { Case reports/series }(\mathrm{N}=181)}{\mathrm{n}(\%)}$} & \multicolumn{3}{|c|}{ Observational studies } \\
\hline & & Number of studies reporting complication & $\mathrm{n} / \mathrm{N}(\%)^{*}$ & 95\% Confidence Interval $(\mathrm{Cl})$ \\
\hline GASTROINTESTINAL & $9(5.0)$ & & & \\
\hline Liver injury & $6(3.3)$ & 3 & $49 / 331(14.8)$ & $11.0-18.6$ \\
\hline Gastrointestinal bleeding & & 1 & $3 / 161(1.9)$ & $0.4-5.4$ \\
\hline Pancreatic injury & & 1 & 9/52 (17.3) & $7.0-27.6$ \\
\hline Ischemic colitis & $1(0.6)$ & & & \\
\hline Hypoproteinemia & & 1 & $36 / 161(22.4)$ & $15.9-28.8$ \\
\hline Acute pancreatitis & $2(1.1)$ & & & \\
\hline RENAL & $23(12.7)$ & & & \\
\hline Renal insufficiency & & 2 & $15 / 253(5.9)$ & $3.3-9.8$ \\
\hline Acute kidney injury & $16(8.8)$ & 3 & $58 / 940(6.2)$ & $4.7-8.0$ \\
\hline Transient acute renal failure & $7(3.9)$ & 1 & $14 / 20(70.0)$ & $49.9-90.1$ \\
\hline DERMATOLOGICAL & $2(1.1)$ & & & \\
\hline Herpes zoster (shingles) & $2(1.1)$ & & & \\
\hline
\end{tabular}

*pooled prevalence of complications in the observational studies expressed as the number of reported complications divided by the total sample size of studies reporting the complication

Table 6: Endocrine, and others complications of COVID-19.

J Microbiol Infect Dis $\quad$ www.jmidonline.org $\quad$ Vol 11, No 2, June 2021




\begin{tabular}{|c|c|c|c|c|}
\hline \multirow{2}{*}{ Complications } & \multirow{2}{*}{$\begin{array}{l}\text { Case reports/series } \\
\qquad \begin{array}{c}(\mathrm{N}=181) \\
\mathrm{n}(\%)\end{array}\end{array}$} & \multicolumn{3}{|c|}{ Observational studies } \\
\hline & & Number of studies reporting complication & $\mathrm{n} / \mathrm{N}(\%)^{*}$ & 95\% Confidence Interval $(\mathrm{Cl})$ \\
\hline ENDOCRINE & $3(1.7)$ & & & \\
\hline Subacute thyroiditis & $1(0.6)$ & & & \\
\hline Diabetic ketoacidosis & $2(1.1)$ & & & \\
\hline OTHERS & $13(7.2)$ & & & \\
\hline $\begin{array}{l}\text { Multiple organ dysfunction } \\
\text { syndrome }\end{array}$ & $2(1.1)$ & 2 & $\begin{array}{l}51 / 253 \\
(20.2)\end{array}$ & $15.2-25.1$ \\
\hline Electrolyte disorders & & 1 & $\begin{array}{c}48 / 161 \\
(29.8)\end{array}$ & $22.8-36.9$ \\
\hline Sepsis & & 1 & $\begin{array}{l}17 / 161 \\
(10.6)\end{array}$ & $5.8-15.3$ \\
\hline Shock & & 1 & $\begin{array}{l}20 / 20 \\
(100)\end{array}$ & 100 \\
\hline Atypical Kawasaki disease & & 1 & $\begin{array}{l}10 / 20 \\
(50.0)\end{array}$ & $28.1-71.9$ \\
\hline Rhabdomyolysis & $11(6.1)$ & 1 & $\begin{array}{c}23 / 214 \\
(10.8)\end{array}$ & $6.6-14.9$ \\
\hline
\end{tabular}

*pooled prevalence of complications in the observational studies expressed as the number of reported complications divided by the total sample size of studies reporting the complication 
The outbreak of COVID-19 is not yet up to a year; the majority of reported complications are within three months of infection. The long-term effects of the infection on the respiratory system are still unknown. Drawing inferences from the sequelae of the 2002 Severe Acute Respiratory Syndrome (SARS) outbreak, the National Health Service, United Kingdom enlists chronic cough, fibrotic lung disease, bronchiectasis, and pulmonary vascular disease as potential longterm complications of COVID-19. Much is still unknown about COVID-19. Therefore, adequate follow-up clinic presentation and chest imaging of COVID-19 pneumonia patients should be recommended in order to detect long-term harbingers of complications and respond promptly $[12,13]$.

\section{Cardiovascular complications}

Pre-existing cardiovascular conditions are established predictors of poor outcomes of SARS-CoV-2 infection. Certain cardiovascular complications have also been associated with COVID-19 [14]. These may result from systemic inflammation, the direct effect of the virus on cardiomyocytes via ACE2 receptors, and increased demand on the heart due to ongoing systemic inflammation $[15,16]$. The mechanisms by which COVID-19 results in cardiovascular complications are not well understood. A likely explanation for the incidence of myocardial infarction is that systemic inflammation causes disruption of atherosclerotic plaques. Moreover, since the activation of ACE2 receptor promotes the excretion of potassium ions, this may result in hypokalemia which puts the heart at risk of arrhythmia [15]. Adverse drug reactions are also potential causes of cardiovascular complications. Hydroxychloroquine with or without azithromycin can disrupt the electrical activity of the heart [17]. Multiple organ dysfunction syndromes (MODS) and shock are associated with severe COVID-19 infection and can eventually lead to death [18].

\section{Hematological complications}

Acute coagulopathy disseminated intravascular coagulation, and deep venous thrombosis were the commonest hematological complications found in this systematic review and metaanalysis (Table 3 ). Previous studies associated sepsis-induced coagulopathy (SIC) and disseminated intravascular coagulation (DIC) with severe COVID-19 cases, especially in nonsurvivors.

\section{Gastrointestinal complications}

From our findings, liver injury was the most frequent gastrointestinal complication (Table 5). Studies reported that autopsies done on COVID19 patients confirmed the presence of the virus in the liver tissues of patients. The underlying mechanisms for hepatic injury in patients with COVID-19 are still not fully understood. However, a study showed that the SARS-CoV-2 receptor ACE2 was highly expressed in bile duct cells. This suggests that SARS-CoV-2 may directly bind to ACE2-positive bile duct cells, leading to liver dysfunction [19]. Another likely explanation for liver injuries associated with COVID-19 is drug hepatotoxicity. Studies reported cases of drug-induced liver injury during treatment of COVID-19 patients with antiviral, antibiotic, and steroid medications. Some patients treated with Remdesivir during the pandemic were also reported to have elevated liver enzymes. Hypoproteinemia was a commonly encountered complication in severe and critical COVID-19 patients; this may have resulted from malnutrition or liver injuries [20].

Bioinformatics analysis revealed that ACE2 was also highly expressed in the glandular cells of gastric and duodenal epithelia. Invasion of the ACE2-expressing enterocytes by SARS-CoV-2 can lead to malabsorption, unbalanced intestinal secretion, activated enteric nervous system, and result in diarrhea. This invasion might explain other gastrointestinal complications, including gastrointestinal bleeding that was reported in this study [21].

\section{Renal complications}

Acute kidney injury was found to be the commonest renal complication; this was followed by renal insufficiency and acute renal failure (Table 5). Researchers found that ACE-2 receptor of SARS-CoV-2 was highly expressed in renal tubules and cells including mesangial cells, podocytes, and parietal epithelium of the Bowman's Capsule, this might be responsible for the renal complications $[22,23]$.

Although mechanisms for the renal manifestations of COVID-19 are still not fully understood, a study proposed a complex multifactorial pathway which includes the 
following: (i) direct viral involvement and replication in the kidneys leading to dysfunction; (ii) local disruption in renin-angiotensinaldosterone system homeostasis (iii) lung protective fluid management strategy during treatment of ARDS and (iv) as a result of a systemic inflammatory response "cytokine storm" [23]. A recent study also demonstrated that the SARS-CoV-2 virus can directly infect human renal tubules consequently leading to acute renal tubular injury [23].

Increased incidence of venous thromboembolic events was also reported in critically ill COVID19 patients admitted to the intensive care unit [24].

COVID-19 infection has been associated with coagulation abnormalities characterized by the elevation of coagulation biomarkers. There were reports of increases in procoagulant factor levels including fibrinogen and increases in D-dimers in COVID-19 patients. This reflects an inflammatory status characterized by coagulation activation and endothelial dysfunction and has been described to be a predictor of death associated with high mortality [24]. The expression of SARS-CoV-2 receptor ACE2 on venous and arterial endothelium is probably responsible for vascular and hematological complications [7].

\section{Neurological complications}

There are few observational studies that assess neurological complications of COVID-19. Cerebrovascular accidents and Guillain-Barre syndrome are mostly reported in case reports/series. There are gaps in the understanding of the neurotropism of SARSCoV-2. Notwithstanding, possible routes of SARS-CoV-2 neuro-invasion have been proposed involving the olfactory epithelium, trans-synaptic spread through peripheral nerves, and lymphatic or hematogenous transfer [25].

\section{Dermatological complications}

Dermatological complications of COVID-19 were reported in just a few case reports/series. These complications are either due to direct invasion of the virus or due to a reaction of the immune system of the body [26]. Herpes zoster is one of the skin complications of COVID-19 and was reported in a case report in which there was a reactivation of the varicella-zoster virus in a patient with COVID-19 [27].

\section{Endocrine complications}

Diabetic ketoacidosis (DKA) and subacute thyroiditis are two of the endocrine complications in patients with COVID-19 reported by a few case reports. Subacute thyroiditis is a late complication of COVID-19 that presents with fever, cough, hoarseness, and odynophagia $[28,29]$. DKA, on the other hand, is a hyperglycemic crisis that is due to insulin deficiency. A previous study showed that there is an increased risk of DKA in diabetic patients with COVID-19 [30,31].

\section{Other complications}

Other complications like multiple organ dysfunction syndromes, electrolyte disorders, sepsis, shock, atypical Kawasaki disease, and rhabdomyolysis can also result from COVID-19 infection. MODS is an end-stage disease that includes acute kidney injury, thrombosis, acute cardiac injury, stroke, viral liver injury, pulmonary embolism, atelectasis, etc. [32]. Hypocalcemia, hypokalemia, and hypernatremia are the common electrolyte disorders that can be caused by COVID-19 infection and may be due to gastrointestinal losses by vomiting or diarrhea [33].

This review is limited by the fact that it was conducted at a time the pandemic is still ongoing. As such, newer complications other than those reported may still arise. Also, the exact causes of many complications are not yet fully understood. Further studies will be needed to elucidate these.

\section{Conclusions}

This review identifies systemic complications associated with COVID-19 infection and the prevalence of these complications. Pulmonary complications such as pneumonia, ARDS, amongst others, are the most reported across all study types, with endocrine and neurological complications least reported. The higher prevalence of pulmonary complications further supports existing literature that the respiratory system is the most affected in COVID-19 infections. With an increase in the number of cases worldwide, this study adds to the existing literature on COVID-19 complications and helps 
improve the current knowledge of healthcare professionals of the impact of COVID -19 infections. It buttresses the need to be on the watch for arising complications across all body systems in patients with COVID 19 infections and ensure prompt treatment and care to avoid debilitating effects associated with the complications.

\section{ACKNOWLEDGMENTS}

\section{Availability of data and materials}

Data generated or analyzed during this study are included in this published article, and/or its supplementary information file will be provided by the corresponding author if demanded.

Declaration of Conflicting Interests: The authors declare that they have no conflict of interest.

\section{Funding: Not applicable REFERENCES}

1. Mao L, Wang M, Chen S, et al. Neurological Manifestations of Hospitalized Patients with COVID19 in Wuhan, China: A Retrospective Case Series Study.

https://www.medrxiv.org/content/10.1101/2020.02.22. 20026500v1,

2. Culp WC. Coronavirus Disease 2019. A A Pract 2020; 14: e01218.

3. Pinna $P$, Grewal $P$, Hall JP, et al. Neurological manifestations and COVID-19: Experiences from a tertiary care center at the Frontline. J Neurol Sci. 2020; 415:116969.

4. Zeng J-H, Liu Y-X, Yuan J, et al. First case of COVID-19 complicated with fulminant myocarditis: a case report and insights. Infection 2020 Oct;48(5):773-777.

5. Gupta A, Madhavan M V., Sehgal K, et al. Extrapulmonary manifestations of COVID-19. Nat Med 2020; 26:1017-32.

6. Kohn MA, Senyak J. Sample Size Calculators [website]. UCSF CTSI. 29 April 2021. Available at https://www.sample-size.net/ [Accessed 10 June 2021]

7. Hamming I, Timens W, Bulthuis MLC, Lely AT, Navis GJ, van Goor H. Tissue distribution of ACE2 protein, the functional receptor for SARS coronavirus. A first step in understanding SARS pathogenesis. J Pathol 2004; 203:631-637.

8. World Health Organization. Clinical management of severe acute respiratory infection (SARI) when COVID-19 disease is suspected. Interim guidance. Pediatr Med Rodz 2020, 16 (1): 9-26.
9. Ranieri VM, Rubenfeld GD, Thompson BT, et al. Acute respiratory distress syndrome: The Berlin definition. JAMA 2012; 307: 2526-2533.

10. Li X, Ma X. Acute respiratory failure in COVID-19: Is it "typical" ARDS? Crit Care 2020 May 6;24(1):198.

11. Helleberg M, Steensen M, Arendrup MC. Invasive aspergillosis in patients with severe COVID-19 pneumonia. Clin Microbiol Infect 2021; 27(1):147-148.

12. Fraser $\mathrm{E}$. Long term respiratory complications of Covid-19. BMJ 2020; 370:m3001. https://doi.org/10.1136/bmj.m3001.

13. NHS. Aftercare needs of inpatients recovering from COVID-19. 2020. https://www.cambscommunityservices.nhs.uk/docs/de fault-source/luton-adults-

general/c0388_after_care_needs_of_inpatients_recov ering from covid-19 5 june 2020.pdf

14. Nishiga M, Wang DW, Han Y, Lewis DB, Wu JC. COVID-19 and cardiovascular disease: from basic mechanisms to clinical perspectives. Nat Rev Cardiol 2020; 17:543-58.

15. Long B, Brady WJ, Koyfman A, Gottlieb M. Cardiovascular complications in COVID-19. Am J Emerg Med 2020; 38:1504-1507.

16. Chen L, Li X, Chen M, Feng Y, Xiong C. The ACE2 expression in human heart indicates new potential mechanism of heart injury among patients infected with SARS-CoV-2. Cardiovasc Res 2020; 116:1097-1000.

17. Mercuro NJ, Yen CF, Shim DJ, et al. Risk of QT Interval Prolongation Associated with Use of Hydroxychloroquine with or without Concomitant Azithromycin among Hospitalized Patients Testing Positive for Coronavirus Disease 2019 (COVID-19). JAMA Cardiol 2020; 5: 1036-1041.

18. Fox S, Vashisht R, Siuba M, Dugar S. Evaluation and management of shock in patients with COVID-19. Cleve Clin J 2020. https://doi.org/10.3949/ccjm.87a.ccc052.

19. Hoffmann M, Kleine-Weber H, Krüger N, Müller M, Drosten C, Pöhlmann S. The novel coronavirus 2019 (2019-nCoV) uses the SARS-coronavirus receptor ACE2 and the cellular protease TMPRSS2 for entry into target 2020. https://www.biorxiv.org/content/10.1101/2020.01.31.9 $29042 v 1$.

20. Musa S. Hepatic and gastrointestinal involvement in coronavirus disease 2019 (COVID-19): What do we know till now? Arab J Gastroenterol 2020; 21:3-8. https://doi.org/10.1016/j.ajg.2020.03.002.

21. Zhang $\mathrm{H}$, Kang $\mathrm{Z}$, Gong $\mathrm{H}$, et al. The digestive system is a potential route of 2019-nCov infection: a bioinformatics analysis based on single-cell transcriptomes. $\quad 2020$ https://www.biorxiv.org/content/10.1101/2020.01.30.9 $27806 \mathrm{v} 1$ 
22. Yang F, Shi S, Zhu J, Shi J, Dai K, Chen X. Analysis of 92 deceased patients with COVID-19. J Med Virol 2020;92(11):2511-2515.

23. Kunutsor SK, Laukkanen JA. Renal complications in COVID-19: a systematic review and meta-analysis. Ann Med 2020; 52:1-9.

24. Connors JM, Levy JH. Thromboinflammation and the hypercoagulability of COVID-19. J Thromb Haemost 2020; 18:1559-61.

25. Chen X, Laurent S, Onur OA, et al. A systematic review of neurological symptoms and complications of COVID-19. J Neurol 2020; J Neurol 2021; 268(2):392402.

26. Gavriatopoulou M, Korompoki E, Fotiou D, et al. Organ-specific manifestations of COVID-19 infection. Clin Exp Med 2020 20(4):493-506.

27. Elsaie ML, Nada HA. Herpes Zoster (shingles) complicating the course of COVID19 infection. J Dermatolog Treat 2020; 12;1-3.

28. Bellastella G, Maiorino MI, Esposito K. Endocrine complications of COVID-19: what happens to the thyroid and adrenal glands? J Endocrinol Invest 2020; 43:1169-70.

29. Ruggeri RM. Subacute thyroiditis in a patient infected with SARS-COV-2: an endocrine complication linked to the COVID-19 pandemic 2020:9-11.

30. Palermo NE, Sadhu AR, McDonnell ME. Diabetic Ketoacidosis in COVID-19: unique concerns and considerations. J Clin Endocrinol Metab 2020; 105 (8):dgaa360..

31. Kumar P, Kuchay MS, Mehta Y, Mishra KS. Diabetic ketoacidosis precipitated by COVID-19: A report of two cases and review of literature. Diabetes Metab Syndr 2020; 14(5): 1459-1462.

32. Robba C, Battaglini D, Pelosi P, Rocco PRM. Multiple organ dysfunction in SARS-CoV-2: MODSCoV-2. Expert Rev Respir Med 2020:1-4. https://doi.org/10.1080/17476348.2020.1778470.

33. Lippi G, South AM, Henry BM. Electrolyte imbalances in patients with severe coronavirus disease 2019 (COVID-19). Ann Clin Biochem 2020; 57:262-265. 